\title{
Integration techniques for surface $X$-ray diffraction data obtained with a two-dimensional detector
}

\author{
Jakub Drnec, Tao Zhou, Stelian Pintea, Willem Onderwaater, Elias Vlieg, \\ Gilles Renaud and Roberto Felici
}

J. Appl. Cryst. (2014). 47, 365-377

Copyright (C) International Union of Crystallography

Author(s) of this paper may load this reprint on their own web site or institutional repository provided that this cover page is retained. Republication of this article or its storage in electronic databases other than as specified above is not permitted without prior permission in writing from the IUCr.

For further information see http://journals.iucr.org/services/authorrights.html

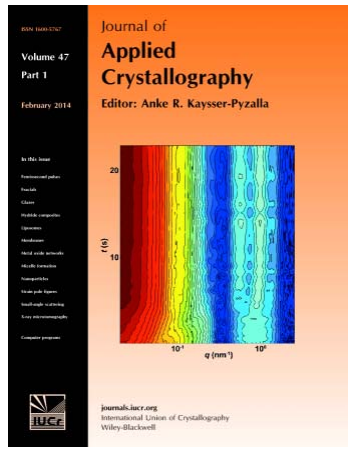

Many research topics in condensed matter research, materials science and the life sciences make use of crystallographic methods to study crystalline and non-crystalline matter with neutrons, X-rays and electrons. Articles published in the Journal of Applied Crystallography focus on these methods and their use in identifying structural and diffusioncontrolled phase transformations, structure-property relationships, structural changes of defects, interfaces and surfaces, etc. Developments of instrumentation and crystallographic apparatus, theory and interpretation, numerical analysis and other related subjects are also covered. The journal is the primary place where crystallographic computer program information is published.

Crystallography Journals Online is available from journals.iucr.org 
Journal of

Applied

Crystallography

ISSN 1600-5767

Received 14 August 2013

Accepted 27 November 2013

\section{Integration techniques for surface X-ray diffraction data obtained with a two-dimensional detector}

\author{
Jakub Drnec, ${ }^{\mathrm{a} *}$ Tao Zhou, ${ }^{\mathrm{b}}$ Stelian Pintea, ${ }^{\mathrm{a}, \mathrm{c}}$ Willem Onderwaater, ${ }^{\mathrm{a}}$ Elias Vlieg, \\ Gilles Renaud $^{b}$ and Roberto Felici ${ }^{a}$
}

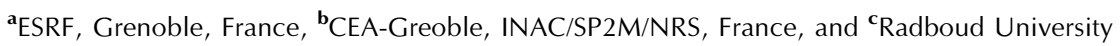
Nijmegen, Institute for Molecules and Materials, Nijmegen, The Netherlands. Correspondence e-mail: drnec@esrf.fr

\begin{abstract}
This article proposes two integration methods to determine the structure factors along a surface diffraction rod measured with a two-dimensional detector. The first method applies the classic way of calculating integrated intensities in angular space. This is adapted to work efficiently with two-dimensional data. The second method is based on integration in reciprocal space. An intensity map is created by converting the detected intensity pixel by pixel to the reciprocal space. The integration is then performed directly on this map. A theoretical framework, as well as a comparison between the two integration methods, is provided.
\end{abstract}

and/or by detector size without additional spatial resolution. Rocking scans need to be performed in order to obtain the integrated intensity of a particular reflection. (During a rocking scan the detector is held fixed while the sample is rotated around a certain axis, often chosen to be the surface normal in SXRD experiments.) In general, two-dimensional detectors have a much larger angular acceptance, and their spatial resolution is defined by their pixel size. Therefore, it is possible to obtain an image of the intensity distribution around a particular reflection in one single acquisition, provided that the detector acceptance is large enough to accept the full size of the reflection. In most cases sufficient information about the peak profile is stored in one image, and therefore structure factor determination is possible without performing a rocking scan (Specht \& Walker, 1993; Vlieg, 1997). This greatly shortens the acquisition time (up to 100 times). Even when the use of rocking scans becomes inevitable (e.g. the acceptance of the two-dimensional detector is not sufficient, which is often the case for lower outgoing angles), a wide range of data along the rod can be extracted thanks to the large volume of $Q$ space covered during the scan.

In both cases (single acquisition and rocking scans), one could choose to reconstruct an intensity map in the reciprocal space and perform the integration directly. A distinct advantage of this newly proposed approach is that it can be applied indiscriminately to (a combination of) any type of scan. An analysis of this concept and its relation to integrated intensities obtained using a point detector will be provided in this article.

\section{Prerequisites}

Unless otherwise specified, the diffraction geometry as well as the associated correction factors described in this article are those of a $Z$-axis (six-circle) diffractometer. The measuring 
techniques are available for all kinds of geometries, though they might bear different names, and the analysis methods can be easily adjusted to yield similar results.

The convention for a six-circle diffractometer (Vlieg, 1997) (Fig. 1) is that $\beta_{\text {in }}$ is the incidence angle and $\beta_{\text {out }}$ the outgoing angle. $\gamma$ is the out-of-plane (of the sample) detector angle, $\delta$ is the in-plane (of the sample) detector angle, and $\omega$ is the sample rotation around the axis perpendicular to the surface or sample azimuth. In the $z$-axis mode, $\beta_{\text {in }}=\alpha$ and $\beta_{\text {out }}=\gamma \cdot h$ and $k$ are the in-plane diffraction indices and $l$ is the out-ofplane diffraction index (often perpendicular to the surface). $\mathbf{Q}$ is the momentum transfer, the difference between the outgoing and the incoming wavevector $\mathbf{Q}=\mathbf{K}_{\mathrm{i}}-\mathbf{K}_{\mathrm{f}}$. This is often defined as the combination of its in-plane and out-ofplane components, $\mathbf{Q}_{\|}=\mathbf{Q}_{X}+\mathbf{Q}_{Y}$ and $\mathbf{Q}_{\perp}=\mathbf{Q}_{Z}$ in the case of surface X-ray diffraction.

\subsection{Traditional integration of data acquired with point detectors}

In the case of off-specular reflections, the integrated intensity of an ideal rocking $(\omega)$ scan (i.e. intensity is recorded

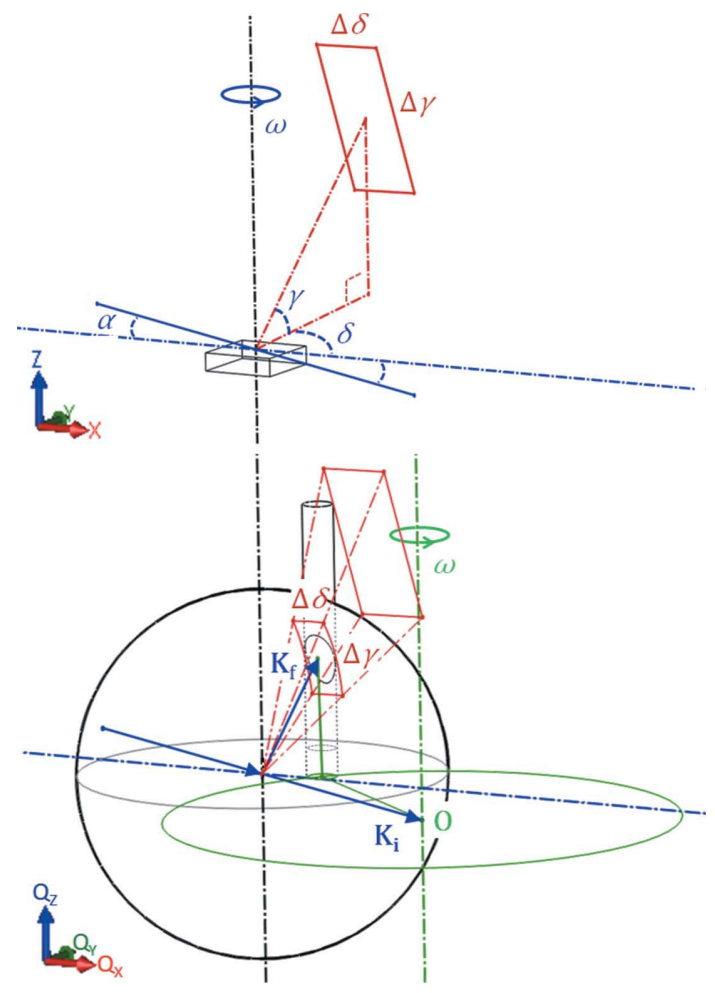

Figure 1

(Top) Graphic representation of the angle convention in real space (in the reference system of the diffractometer). The incident beam is confined in the $X Z$ plane, while the sample surface is parallel to the $X Y$ plane. (Bottom) Graphic representation of the angle convention in reciprocal space. For elastic scattering we have $\left|\mathbf{K}_{\mathrm{i}}\right|=\left|\mathbf{K}_{\mathrm{f}}\right|$. The point $\mathbf{O}$ denotes the reciprocal space origin. A homogeneous crystal truncation rod is added (black cylinder); the intersection with the Ewald sphere is illustrated as a deformed ellipse. The sample surface plane is shown as a grey circle, while the green circle depicts the reciprocal plane $\mathbf{Q}_{\perp}=0$. Also shown is the projected detector acceptance onto the Ewald sphere, given by $K_{\mathrm{f}} \Delta \gamma$ and $K_{\mathrm{f}} \Delta \delta$. while continuously rotating the sample around its surface normal) is (Vlieg, 1997, equations 4 and 5)

$$
I_{\omega, \text { ideal }}=\left(\frac{\Phi_{0}}{\omega_{0}}\right)\left(\frac{A}{A_{\mathrm{u}}}\right) \iiint r_{\mathrm{e}}^{2}\left|F_{h k l}\right|^{2} P u(Q) \mathrm{d} \gamma \mathrm{d} \delta \mathrm{d} \omega,
$$

where $\Phi_{0}$ is the incident flux, $\omega_{0}$ the rotation speed, $r_{\mathrm{e}}$ the classical electron radius, $A$ the active surface area, $A_{\mathrm{u}}$ the area of the surface unit cell, $F_{h k l}$ the structure factor of the $h k l$ reflection, $P$ the polarization factor and $u(Q)$ the line shape function. Usually an $\omega$ scan is discrete and consists of $N_{\omega}$ points distributed along the scan path with an acquisition time of $T_{\omega}$ for each point,

$$
I_{\omega}=\Phi_{0} T_{\omega}\left(\frac{A}{A_{\mathrm{u}}}\right) \sum_{i=1}^{N_{\omega}} \iint r_{\mathrm{e}}^{2}\left|F_{h k l}\right|^{2} P u(Q) \mathrm{d} \gamma \mathrm{d} \delta \Delta \omega_{i} .
$$

$I_{\omega}$ can be eventually rewritten with the following correction factors (Vlieg, 1997, equation 42):

$$
I_{\omega}=\Phi_{0} T_{\omega} \frac{A}{A_{\mathrm{u}}^{2}} r_{\mathrm{e}}^{2} \lambda^{2}\left|F_{h k l}\right|^{2} P C_{\mathrm{area}} C_{\mathrm{det}} C_{\text {beam }} \frac{\Delta \gamma \cos \gamma}{\cos \alpha \sin \delta \cos \gamma} .
$$

Here $C_{\text {area }}$ is the area correction factor, which is proportional to the illuminated surface area and is traditionally defined by the size of the incoming beam parallel to the surface and by the opening of the slits in front of the detector (Vlieg, 1997). $C_{\text {beam }}$ compensates for the intensity distribution profile of the incoming beam. $C_{\mathrm{det}}$ is present in the case where the in-plane acceptance of the detector is not sufficiently large to cover the entire peak. This will be dealt with in $\S 5.1 /(\cos \alpha \sin \delta \cos \gamma)$ is the Lorentz factor for the $\omega$ scan. $\cos \gamma$ is the rod interception factor, which is an integration correction dependent on the geometry of the rod-detector interception, assuming that $\left|F_{h k l}\right|$ is invariant along $l$ within the considered integration volume:

$$
\frac{\partial\left|F_{h k l}\right|}{\partial l} \simeq 0
$$

In the case of a stationary scan, a single data acquisition of duration $T_{\mathrm{s}}$ is carried out at a specific point in the reciprocal space. If we consider the in-plane detector acceptance to be larger than the cross section of the rod and $\left|F_{h k l}\right|$ to be invariant along $l$ within the intersected area, it is reasonable to assume that with one single acquisition we can collect enough data to allow calculation of the rod structure factor at the given $l$ value. The integrated intensity on the detector $I_{\mathrm{s}}$ is

$$
\begin{aligned}
I_{\mathrm{s}} & =\Phi_{0} T_{\mathrm{s}}\left(\frac{A}{A_{\mathrm{u}}}\right) \iint r_{\mathrm{e}}^{2}\left|F_{h k l}\right|^{2} P u(Q) \mathrm{d} \gamma \mathrm{d} \delta \\
& =\Phi_{0} T_{\mathrm{s}} \frac{A}{A_{\mathrm{u}}^{2}} r_{\mathrm{e}}^{2} \lambda^{2}\left|F_{h k l}\right|^{2} P C_{\text {area }} C_{\text {det }, \mathrm{s}} C_{\text {beam }} \frac{1}{\sin \gamma}
\end{aligned}
$$

where $1 / \sin \gamma$ is the Lorentz factor for the stationary measurements (Vlieg, 1997).

As will be shown below, the measuring time can be greatly reduced by combining rocking scans and stationary measurements to cover complementary parts of the rod ( $\$ \$ 3.1$ and 3.2). Concordant results are found in overlapped regions measured by the two techniques (\$3.4). Other approaches include the 
reciprocal space integration, which treats the two types of scans in an identical way, as will be introduced in $\$ 4$.
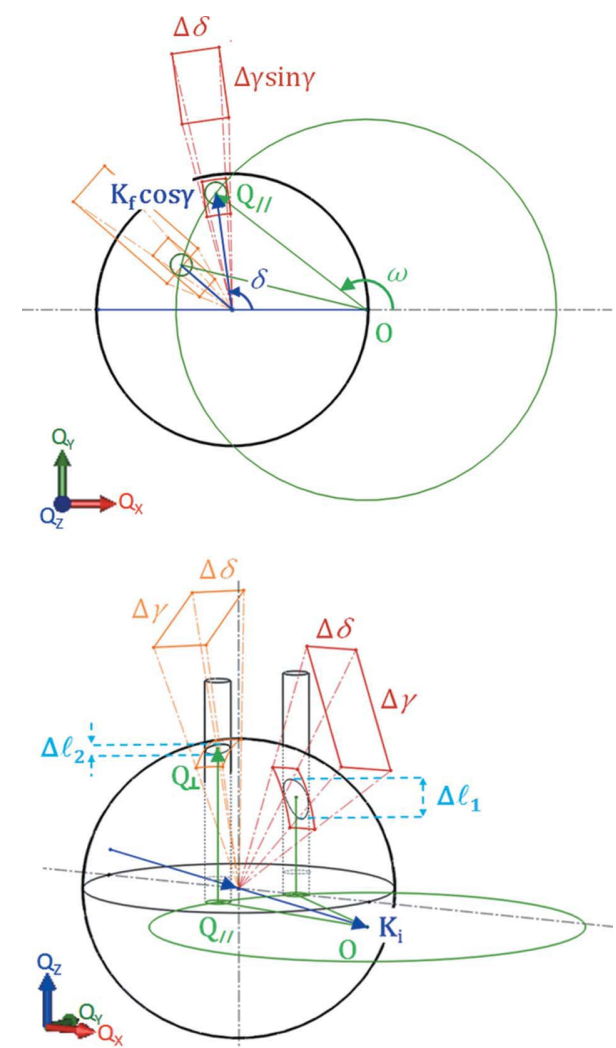

Figure 2

(Top) Top view of two stationary measurements of the same rod at different $l$ values. The in-plane projection of the finite acceptance of the detector is proportional to $\sin \gamma$ and thus is smaller for lower $l$ values (red) than for higher $l$ values (orange). The measurement is only valid if the projected rectangle is large enough to contain the rod cross section (small green circle). (Bottom) Perspective view of the same measurements. Integration should only be carried out if the intensity distribution is approximately uniform along $l$ within the intersected range $\Delta l$, which itself is proportional to $\cos \gamma$. The condition is thus generally easier to fulfill at higher $l$ values (orange) than for lower $l$ values (red).

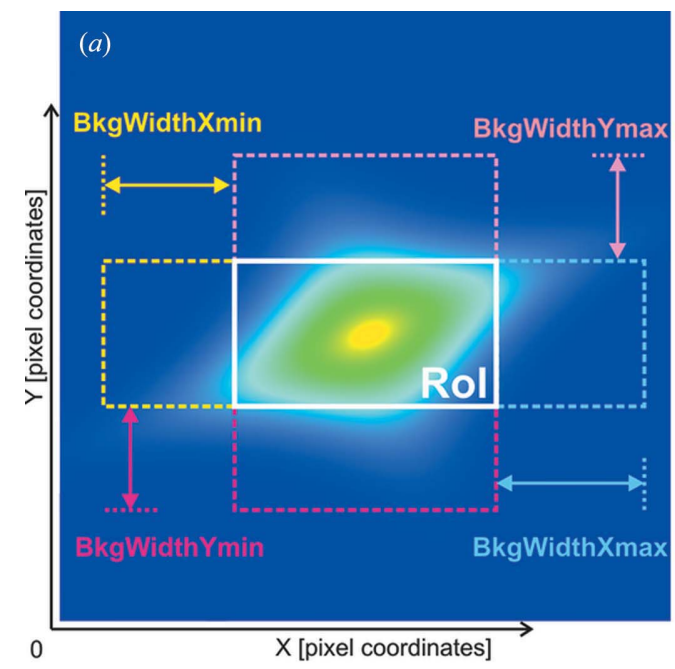

\section{Adapted classic approaches}

\subsection{Stationary scans}

Usually, a complete rod intensity profile is measured through a series of rocking scans at different $l$ values along the rod. By using two-dimensional detectors, it is possible to replace each rocking scan by one single stationary measurement centered on the rod at the same $l$ value, thus vastly speeding up data acquisition. However, certain conditions must be fulfilled (Fig. 2):

(1) The in-plane projection of the finite acceptance of the detector expanded by $K_{\mathrm{f}} \Delta \gamma \sin \gamma$ and $K_{\mathrm{f}} \Delta \delta$ should be sufficiently large to fully include the cross section of the rod (Fig. 2, top). This is not always true as $\sin \gamma$ vanishes at $\gamma=0$. Hence, for low $l$ values, one often has to employ a rocking scan (\$3.2) or attempt to compensate for the missing intensity either analytically or numerically (\$5).

(2) $\left|F_{h k l}\right|$ should be approximately constant over the intersected $l$ range $\Delta l$ (Fig. 2 , bottom).

For proper estimation of the background intensity, the field of view of the detector should be large enough to fully intersect the rod and accept an area with background intensity. Over (or under)-estimation of the background may result in significant errors in the calculated structure factors. A quick and straightforward approach is to first calculate the overall integrated intensity inside a region of interest (RoI) covering the intercepted rod and then subtract the background intensity taken elsewhere (Fig. 3).

During the stationary scan, the post sample slits are often wide open in order to fully accept the rod profile. As a result, intruder signals (signals coming from outside the sample) may be found in close vicinity to the peak or even overlapping the peak, making it impossible to isolate the peak from the background with a regularly shaped RoI (e.g. rectangle). Applying an irregularly shaped RoI (e.g. a polygon) can solve the problem but requires the RoI to be determined for each image as the peak shape evolves during the scan (see Fig. 5 below). An alternative is to use peak shape determination and

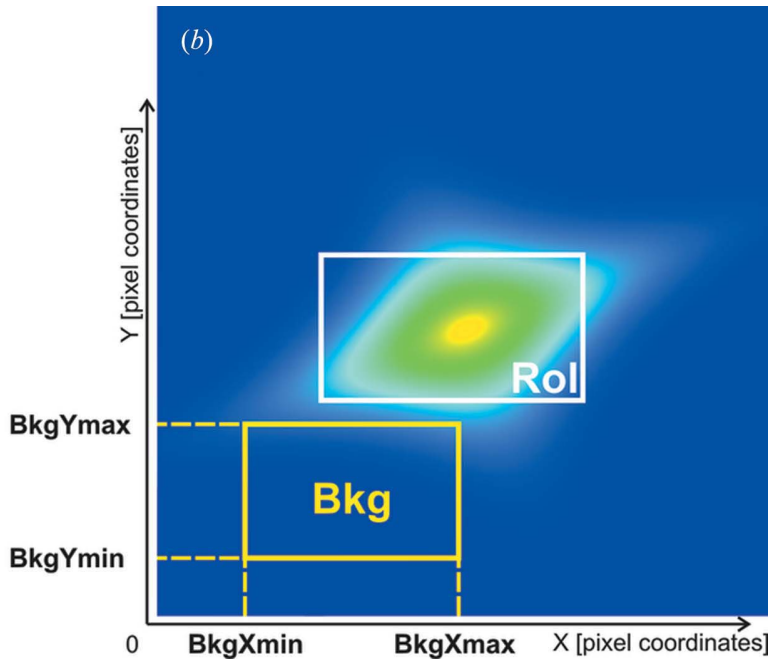

Figure 3

Different ways of selecting the background: $(a)$ rectangles adjacent to each RoI edge; $(b)$ rectangular area outside the RoI. 
background subtraction employing a two-dimensional peak search algorithm. Here we apply the 'seed skewness' method described by Sjölin \& Wlodawer (1981). Because the size of the acquired peak profile is dependent on $\sin ^{-1} \gamma$ (the Lorentz factor) and the center of the peak is not necessarily at the same position for each image taken during the scan, a new peak search has to be performed for each individual image in order to correctly locate the peak area. The background is estimated with the following procedure. (1) For each row of pixels corresponding to the same $\mathbf{Q}_{\perp}$ value in the reciprocal space (along the $\delta$ direction), the background intensities inside the peak contour are estimated with a normal, Gaussian, distribution. (2) The parameters of the normal distribution are calculated using pixels belonging to this row but located outside the peak contour within a specific range (less than five pixels away from the peak contour). (3) The background intensities for each pixel within the peak contour of this row are then computed numerically with a random normal distribution generator. (4) Finally, the background intensity is subtracted pixel by pixel before integration.

Fig. 4 shows a simple demonstration of the robustness of the algorithm when a peak with a very low noise level is considered. The blue rectangle depicts the RoI for the peak search. (P1) The black contour indicates the peak area identified by the peak search routine. (B1) The intensities within the peak contour are replaced by the background intensities found with the normal distribution generator. These are then subtracted during the structure factor calculation. (P2) To further illustrate the performance of the background estimation, we manually impose a smaller peak region. (B2) The calculated background matches the expected background intensities very well in the peak region. (P3) A even smaller peak region is imposed. (B3) Again, the algorithm-estimated background is in agreement with the expected background. (P4) The peak search algorithm performs equally well under a nonzero uniform background which was generated by increasing the count of each pixel by a certain value. The size of the peak is very close to that in $(\mathrm{P} 1)$ and the uniform background is correctly estimated (B4). (P5) To address a more complex problem, an artificial rectangular-shaped background is added to the existing image in order to simulate diffuse intruder signals coming from outside of the sample. The intense rectangular-shaped background is now included in the peak contour as the altered background intensity is higher than the minimum peak intensity. (B5) This does not affect the calculation of the structure factor, owing to the correct estimation of the background intensities in the overlapping area. It should be noted that this algorithm correctly estimates background in most cases, such as a linear or a slowly sloping background. However, caution needs to be taken when the background is a step function or a fast changing nonlinear function in the in-plane direction ( $\delta$ direction in Fig. 4). Apparent vertical streaks in the estimated background images are an artifact of the row-by-row background determination, already discussed in the previous paragraph.

Because the lower range of the peak pixel intensities is the same as the higher range of the background pixel intensities, it is impossible to find the exact boundary between the peak and the background areas. Hence, a way of varying the peak contour in a reasonable manner should be implemented to allow the user to decide which contour is optimal (Sjölin \& Wlodawer, 1981). In our case, we have implemented an
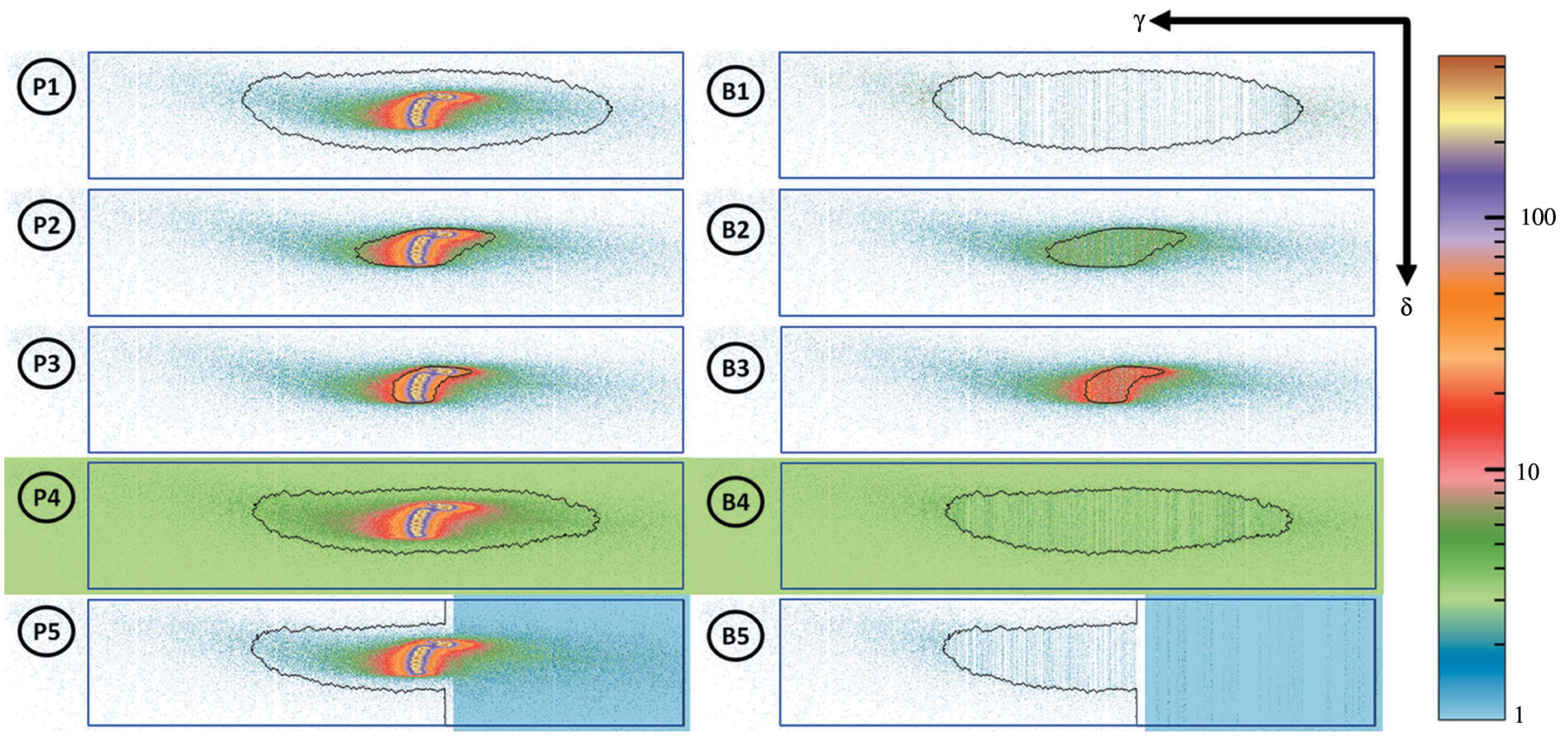

Figure 4

A demonstration of the robustness of the peak search algorithm. The intensity is illustrated by false color levels and is in logarithmic scale. In each case a peak search is performed inside the rectangular-shaped RoI, followed by a background estimation within the same region. The resulting peak is indicated by the black contour. On the left: the peak found on the original, unmodified image. On the right: the background estimated within the peak contour to be subtracted pixel by pixel before the final integration. 
adjustment function on the peak contour using binary morphology techniques such as binary closing, dilation, erosion and opening (Serra, 1982). It is worth mentioning that, since the dominating intensities are always included in the peak region, a reasonable adjustment of the peak contour has a limited, yet visible, effect on the calculation of the structure factor.

The complete analysis procedure of stationary $l$ scans, depicted schematically in Fig. 5, is the following:

(1) For a given $(h, k)$ rod, raw data are collected by conducting stationary measurements at different $l$ values $(\gamma$ positions). Note that the position of the measured peak remains fixed in the detector frame, while its width is stretched by the Lorentz factor $\sin ^{-1} \gamma$. The shape of the peak is also slightly rotated as a result of the change in $\gamma$ and $\delta$ angles of the detector.

(2) For each image a peak search is performed in order to locate the peak area, which gradually evolves during the scan.

(3) The background intensities within the peak area are estimated and subtracted.

(4) Finally, the correction factors are applied to the integrated intensity (see $\S 2$ ). The entire data set is assembled to compute the $\left|F_{h k l}\right|$ profile of the rod.

\subsection{Rocking scans}

A significant part of the rod is measured when performing a rocking scan, owing to the nonzero out-of-plane acceptance of the two-dimensional detector (Fig. 6, bottom). The 'height', $\Delta l$, of this intercepted volume in the $l$ direction is given by $K_{\mathrm{f}} \Delta \gamma \cos \gamma$, which is $K_{\mathrm{f}} \Delta \gamma$ when the detector approaches the surface plane $(\cos \gamma \simeq 1)$. The classic approach for a point detector is to treat the entire rocking scan for one $l$ value as a whole as there is no spatial resolution. In the case of a twodimensional detector, this would correspond to measurement with sufficiently closed detector slits or/and use of only one region of interest during the integration of rocking scan images, yielding just one structure factor for one rocking scan at one given $l$ value. This is both inefficient and inaccurate because, for large out-of-plane acceptance $\Delta \gamma$, equation (4) may not be fulfilled. A more appropriate approach consists of treating data from different images that share the same $l \pm \delta l / 2$ value together. These data, when combined to form new images, give access to consecutive cross sections of the diffraction rod, hence allowing us to extract several structure factors within the entire intercepted $\Delta l$ range. (Fig. 7). The subsequent treatment consists of a peak search on the newly formed images, background subtraction, integration and application of the correction factors. It should be noted that in this case the correction factors [equation (3)] are different from those used for a stationary scan [equation (5)].

The complete analysis procedure of the rocking scans, schematically depicted in Fig. 7, is the following:

(1) For a given $(h, k)$ rod, raw data are collected by performing an $\omega \operatorname{scan}(\mathrm{s})$ at one (or multiple) $l$ value(s). Note

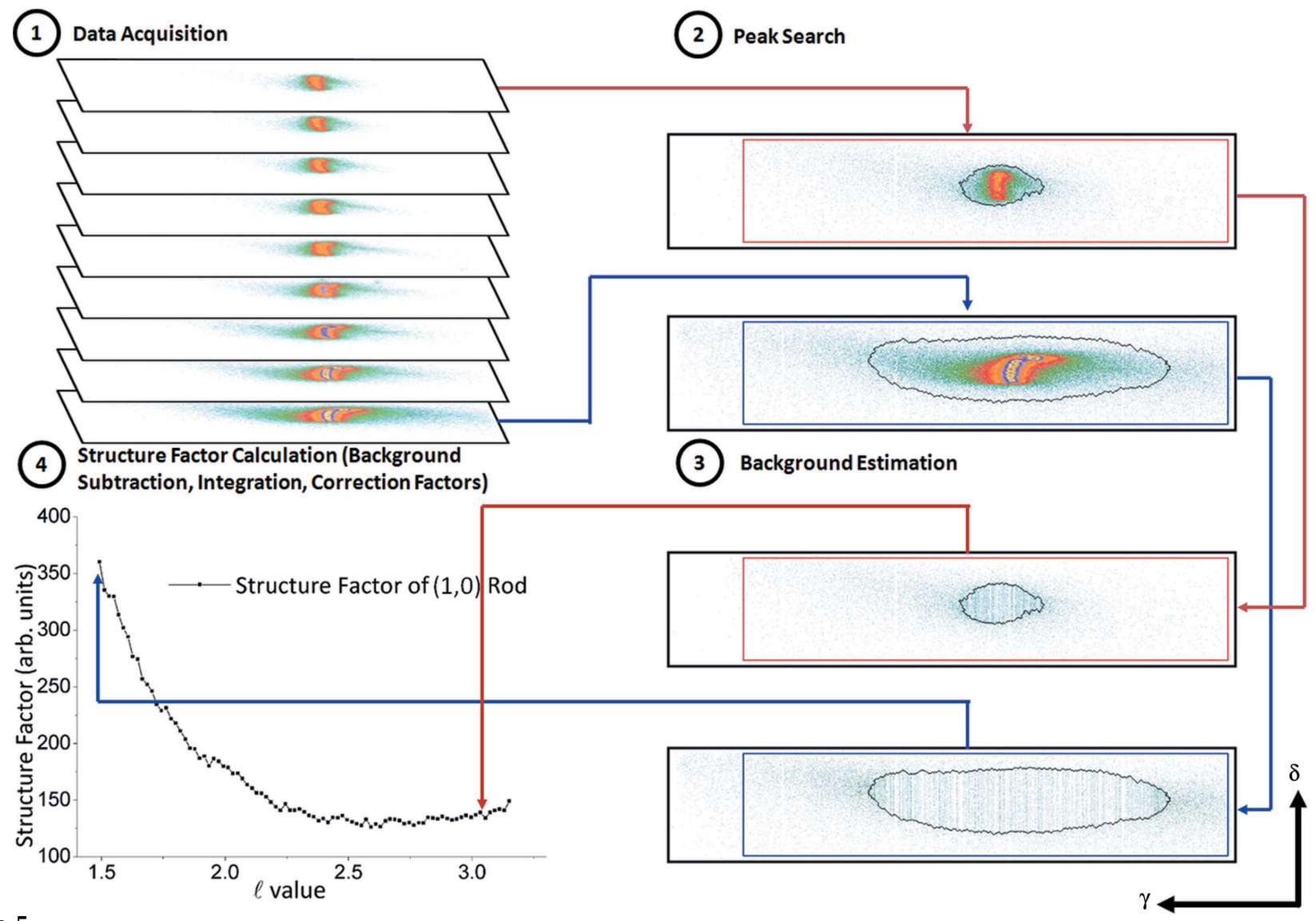

Figure 5

Complete analysis procedure of stationary $l$ scans. The example used here is a $(1,0, l)$ scan of an $\operatorname{Ir}(111)$ sample. 
that the size of the peak remains practically unchanged while its center drifts within the detector frame during the scan.

(2) For each scan, pixels with the same $l$ values $\left(l_{i}, l_{j}, \ldots\right)$ are regrouped to form a new image. This yields a series of crosssectional views of the rod at different $l$ values. Normally, instead of generating one new image for every possible $l$ value, the two-dimensional image data are divided into a number of slices with thickness $\Delta l$. A simple summation along the $\gamma$ direction is then applied to the pixels that fall into $\left(l_{i} \pm \Delta l / 2\right)$ to increase the statistical power of each slice. The result of this operation is a series of newly formed images lying in the $\delta-\omega$ plane.

(3) A peak search is then performed on each newly created $\delta-\omega$ image to locate the peak area. The background intensities are estimated within the peak area in the same way as in the case of a stationary scan.

(4) Finally, the correction factors are applied to the integrated intensity (see $\$ 2$ ). The entire data set is assembled to compute the $\left|F_{h k l}\right|$ profile of the rod.

\subsection{Uncertainty}

For each calculated structure factor, the associated statistical error has to be estimated, for structure factors calculated

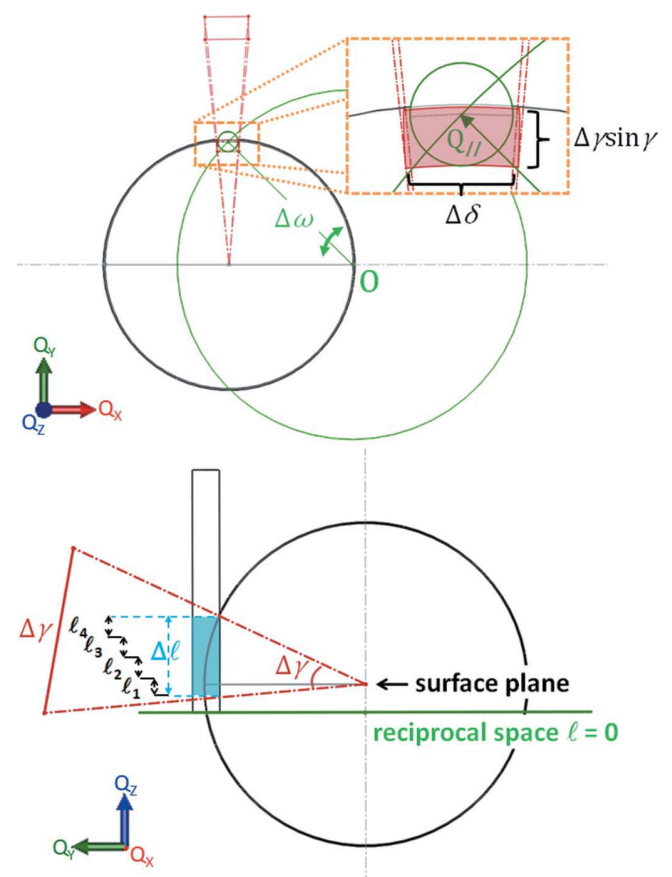

Figure 6

(Top) Top view of one measuring point during a rocking scan at low outgoing angle $\gamma$. The in-plane projection of the detector acceptance (red filled rectangle), being proportional to $\sin \gamma$, no longer contains the entire cross section of the surface rod (small green circle). A full integration thus requires a series of measurements spread along $\Delta \omega$, so that $Q_{\|} \Delta \omega \gg 2 \mathrm{FWHM}$ of the rod. In reality $\Delta \omega$ should be much larger to take into account the inclination of the detector and to measure background intensities. (Bottom) Side view of the same measuring point. To increase measurement efficiency, we take advantage of the nonzero out-of-plane detector acceptance $\Delta \gamma$. By carefully rearranging the data acquired with the two-dimensional detector and regrouping those that correspond to the same $l$ values $\left(l_{1}, l_{2}, \ldots\right)$, it is possible to reconstruct the rod intensity profile within the $\Delta l$ range. both by directly integrating data acquired in one stationary measurement and by integrating cross-sectional images generated from a rocking scan. The statistical error for a linear background is (Robinson, 1990)

$$
\sigma_{i}=\left(I_{i, \mathrm{~S}}+\frac{N_{i, \mathrm{~S}}}{N_{i, \mathrm{~B}}} I_{i, \mathrm{~B}}\right)^{1 / 2},
$$

where $I_{i, \mathrm{~S}}$ is the integrated measured intensity within the peak area, $N_{i, \mathrm{~S}}$ is the number of data points (in pixel counts) in the peak area, $N_{i, \mathrm{~B}}$ is the number of data points used for background estimation and $I_{i, \mathrm{~B}}$ is the integrated background intensity of these points. The statistical errors of the same equivalent reflections $\left(j_{1}, j_{2}, \ldots, j_{n}\right)$ are then used to find the weighted statistical error of the group $\sigma_{j, \text { stat }}$, which when combined with the overall systematic error $\varepsilon$ is often used as a fair estimation of the uncertainty $\sigma_{j}$ of the calculations (Vlieg, 2012):

$$
\begin{aligned}
\sigma_{j, \text { stat }} & =1 /\left(\sum_{k=1}^{n} 1 / \sigma_{j_{k}}\right)^{1 / 2}, \\
\sigma_{j} & =\left(\sigma_{j, \text { stat }}^{2}+\varepsilon^{2}\right)^{1 / 2} .
\end{aligned}
$$

As previously mentioned, increasing the 'thickness' $\Delta l$ when treating two-dimensional rocking scan data has the effect of increasing the statistical significance of each slice. This is illustrated in Fig. 8 (left), where the same rocking scan data are analyzed by choosing two different $\Delta l$ values. Having a larger $\Delta l$ increases the raw integrated intensity of each slice, which leads to a smaller statistical error, as evident from equation (6).

\subsection{Comparison between the two approaches}

Rocking scans are suitable candidates for replacing stationary measurements at low $l$ values. When analyzed properly, the two methods can indeed act complementarily (Fig. 8, right), leading to faster data acquisition and analysis time.

\subsection{Divergence problem}

The divergence problem may be one of the biggest concerns when using two-dimensional detectors with open post sample slits (Mariager et al., 2009). This problem arises from the fact that each pixel of the detector detects photons scattered from the entire illuminated area at different scattering angles (Fig. 9). The effect is more pronounced when the incoming beam is wide and/or when working at low incidence angle (large incoming beam footprint).

As a result, the measured signal is a combination of scattering from the border and the center of the sample. This can make interpretation more difficult if the sample is not homogeneous and/or has a border that scatters very differently from the center of the sample. One can certainly choose to limit the detector's field of view by adding a pair (or multiple pairs) of anti-diffusion slits, but this does not solve the divergence problem completely. Besides, closing the slits restricts the scattered signal to just a small part of the detector, 
which in turn costs us the advantage of using a large area of the pixel array. Another disadvantage of adding post sample slits is that the area correction factor for each pixel needs to be calculated independently since the area seen by each pixel is no longer determined by the incident beam's half-width and its profile but also by the position and opening of the slits. It is worth mentioning that the divergence problem will not significantly affect the integrated intensity in some specific situations, such as when the peak cross section is smaller than the detector acceptance and the intensity profile of the rod is not changing rapidly in the $l$ direction.

A possible solution to the divergence problem is to use converging Soller slits. In this case, each pixel detects photons scattered along specific directions from a limited surface area close to the sample center. This also resolves the border issue. In practice, instead of having ideal two-dimensional converging Soller slits one could use a set of two one-dimensional slits positioned perpendicular to each other (Fig. 10). This has

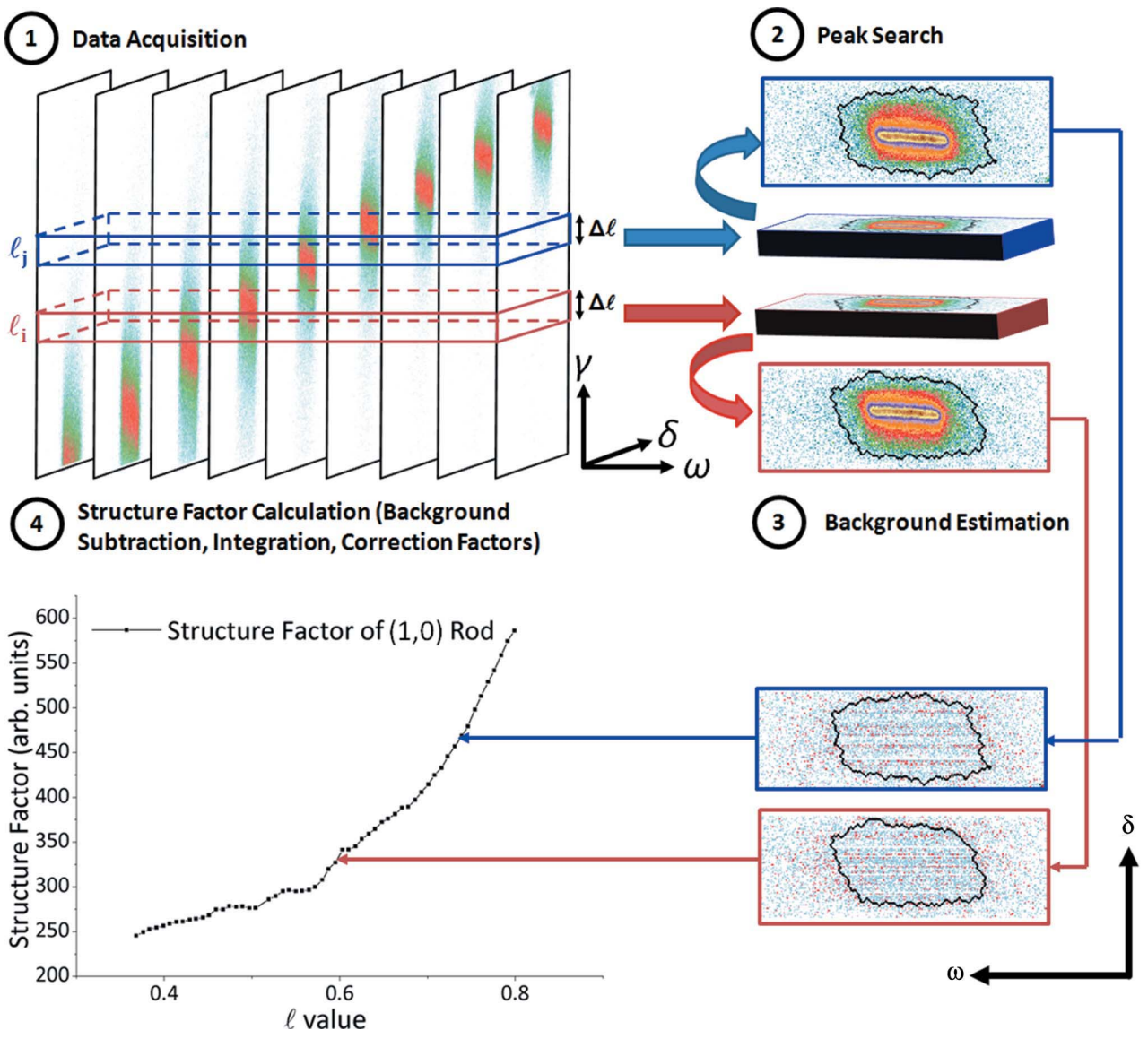

Figure 7

Complete analysis procedure of rocking scans using two-dimensional detectors. The example used here is a rocking scan at $l=0.6$ of the $\operatorname{Ir}(111)(1,0)$ rod.

Figure 8
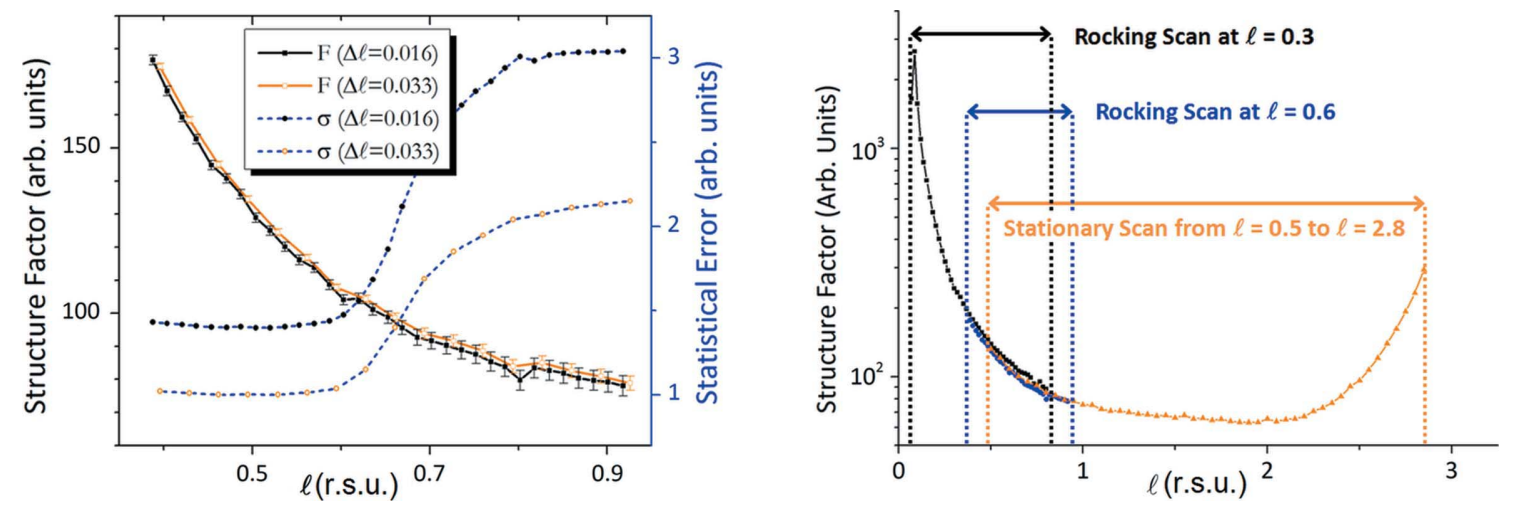

(Left) Treating rocking scan data with two different slice thicknesses $\Delta l$. Solid lines: calculated structure factors with statistical error. Dashed lines: the statistical error alone. (Right) A demonstration of the combination of the two techniques. Two rocking scans cover $l$ ranging from 0.03 to 0.8 and a stationary measurement covers $l$ from 0.5 to 2.8. The two techniques yield concordant results in the overlapping regions. 
the advantage of allowing enhancement of the signal by removing one of the slits when divergence in that direction can be overlooked. Further analysis will be provided in a future publication.

\section{Reciprocal space integration}

In the previous section we analyzed integration methods already approached by other authors. This part of the article proposes a new method to integrate images obtained with a two-dimensional detector, based on the projection of measured data in the reciprocal space. The main advantage of this method is that detector images from rocking scans, stationary $l$ scans or any other kind of scan can be merged and analyzed together. Subsequently the structure factors $F_{h k l}$ can be precisely determined regardless of the diffraction geometry and without using extra parameters often determined experimentally. The technique is based on integration in reciprocal space rather than integration in the angular space of the diffractometer. The only requirement is the knowledge of the matrix converting the diffractometer angles to $h, k, l$ values. This is used to convert the intensity acquired in each pixel of each two-dimensional detector image taken during the scan to the reciprocal space intensity map (voxelization). The integration can then be realized when the map is constructed. One of the advantages is a better statistical error obtained by averaging data taken for the same volume of reciprocal space during the scan (in the case of an image overlap).

The integrated intensity of a given volume in the angular space of the detector is [Vlieg, 1997, equation (47); Cowley, 1975; Vlieg \& Robinson, 1992]

$$
I_{\text {int, } \mathrm{a}}=\Phi_{0} T_{\mathrm{r}} \iiint \frac{\mathrm{d} \sigma}{\mathrm{d} \Omega}(\mathbf{Q}) \mathrm{d} \gamma \mathrm{d} \psi \mathrm{d} \varphi,
$$

where $\Phi_{0}$ is the incident flux, $T_{\mathrm{r}}$ is the counting time, $(\mathrm{d} \sigma / \mathrm{d} \Omega)(\mathbf{Q})$ is the differential cross section and $\mathrm{d} \gamma \mathrm{d} \psi \mathrm{d} \varphi$ is the integration volume. While the integration volume is in the

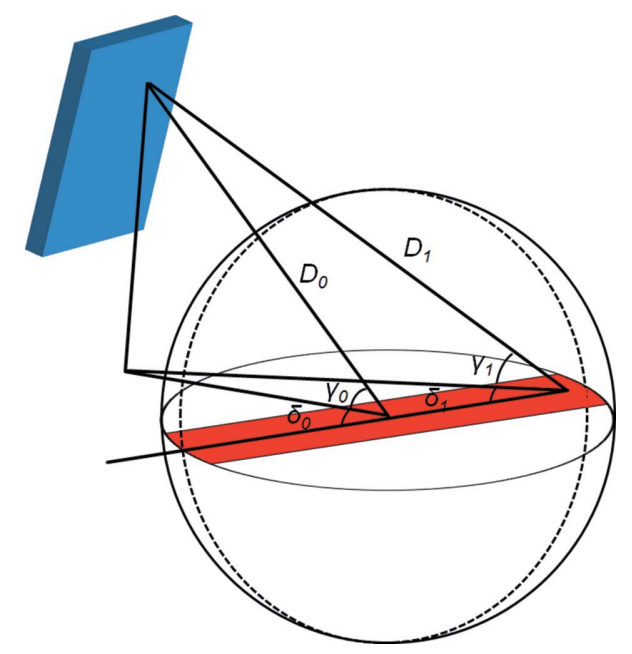

Figure 9

Graphic representation of the divergence problem. Each pixel in the twodimensional detector detects photons scattered from a different part of the sample at different scattering angles, both in plane and out of plane. angular space of the detector, the differential cross section is expressed in terms of the diffraction vector $\mathbf{Q}$ and the integration must be performed in reciprocal space.

The Lorentz correction, $L$, accounts for the angular to reciprocal space volume transformation (Vlieg, 1997), $\mathrm{d} \gamma \mathrm{d} \psi \mathrm{d} \varphi=\left(\lambda^{3} / V_{\mathrm{u}}\right) L \mathrm{~d} h \mathrm{~d} k \mathrm{~d} l$. If we integrate directly in reciprocal space, the integrated intensity is

$$
I_{\mathrm{int}, \mathrm{r}}=\Phi_{0} T_{\mathrm{r}} \iiint \frac{\mathrm{d} \sigma}{\mathrm{d} \Omega}(\mathbf{Q}) \mathrm{d} h \mathrm{~d} k \mathrm{~d} l
$$

and

$$
I_{\mathrm{int}, \mathrm{a}}=\frac{\lambda^{3}}{V_{\mathrm{u}}} L I_{\mathrm{int}, \mathrm{r}},
$$

where $V_{\mathrm{u}}$ is the volume of the unit cell. For stationary scans

$$
I_{\mathrm{int}, \mathrm{a}}=\frac{\lambda^{2}}{A_{\mathrm{u}}} L I_{\mathrm{int}, \mathrm{r}},
$$

where $A_{\mathrm{u}}$ is the area of the surface unit cell. In fact, the Lorentz correction is included when the pixel intensities of two-dimensional images are converted to the three-dimensional reciprocal space intensity map.

To find the surface structure, we need to determine the structure factors from intensities measured along diffraction rods (Robinson \& Tweet, 1992; Feidenhans'1, 1989). Normally, such integrated intensities depend on the detector acceptance. The range in $l$ direction, $\Delta l$, measured by a detector is not a constant and varies with the component of the scattering vector perpendicular to the surface $\left(\mathbf{Q}_{\perp}\right)$ and the $\gamma$ angle in the angular space of a detector. This is taken into account with the $\cos \gamma$ rod interception factor in equation (3). However, if the integration is done directly in reciprocal space, $\Delta l$ can be chosen to be the same for each $l$ value given that the reciprocal space map has enough data points. Assuming that $\Delta l$ is small enough so that $(\mathrm{d} \sigma / \mathrm{d} \Omega)(\mathbf{Q})$ is constant within $\Delta l$ we can write

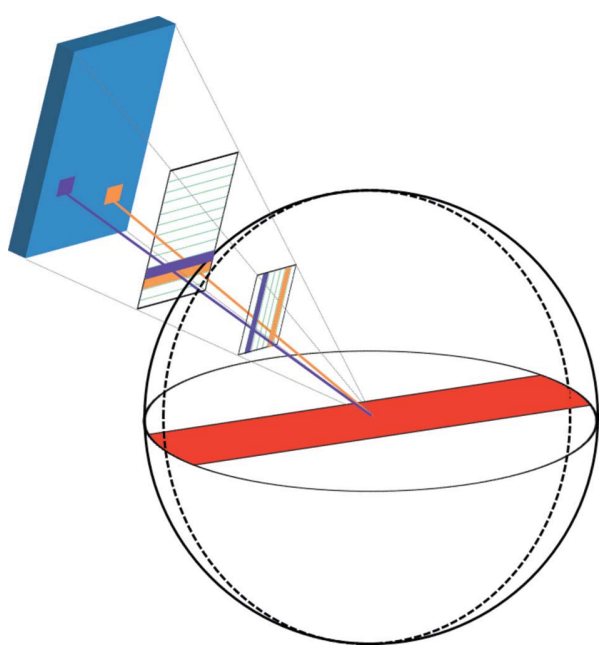

Figure 10

The use of two one-dimensional slits positioned perpendicular to each other to solve the divergence problem and the border issue. 


$$
\begin{aligned}
I_{\text {int, } \mathrm{r}} & =\Phi_{0} T_{\mathrm{r}} \iiint \frac{\mathrm{d} \sigma}{\mathrm{d} \Omega}(\mathbf{Q}) \mathrm{d} h \mathrm{~d} k \mathrm{~d} l \\
& =\Phi_{0} T_{\mathrm{r}} \Delta l \iint \frac{\mathrm{d} \sigma}{\mathrm{d} \Omega}(\mathbf{Q}) \mathrm{d} h \mathrm{~d} k .
\end{aligned}
$$

Substituting for $\iint(\mathrm{d} \sigma / \mathrm{d} \Omega)(\mathbf{Q}) \mathrm{d} h \mathrm{~d} k$ following Vlieg (1997), we get

$$
I_{\text {int }, \mathrm{r}}=\Phi_{0} T_{\mathrm{r}} \frac{A}{A_{\mathrm{u}}} r_{\mathrm{e}}^{2}\left|F_{h k l}\right|^{2} P C_{\text {area }} C_{\text {beam }} \Delta l .
$$

In this case $\Delta l$ is a constant. The $C_{\text {det }}$ correction is omitted because, as will be shown in the next section, it is possible to recover the in-plane shape and intensity distribution of the rod even if the post sample slit width is smaller than the width of the reflection at the camera distance. The choice of $\Delta l$ and the determination of the intensity within $\Delta l$ will be discussed in the example.

\subsection{Reciprocal space integration - example}

In this section, we will show an example of direct integration in reciprocal space and explain all of the important steps. This approach is suitable in cases where diffraction rods are too wide to fit the detector or accurate $F_{h k l}$ values at low $l$ are needed. To get the reciprocal space intensity map, it is necessary to perform scans that sufficiently sample the desired volume of reciprocal space. In this example we use a rocking scan for each $l$, as this can be easily implemented for all diffractometer geometries. However, other scan directions are possible and, in fact, it has been shown that the optimal sampling can be achieved with scans in the directions perpendicular to the Ewald sphere (along the $\mathbf{K}_{\mathrm{f}}$ directions) (Mariager et al., 2009; Schlepütz et al., 2011). These scans can be realized by changing the incoming beam energy or by moving two or more motors simultaneously. This is not always practical and may be difficult to implement on some experimental setups.

Pixels from all images measured during the rocking scans are assigned $h, k, l$ coordinates from the angles $\gamma$ and $\delta$ calculated using the $\gamma_{0}$ and $\delta_{0}$ angles of the central pixel, the pixel size and the detector-sample distance (Schlepütz et al., 2011; Kriegner et al., 2013). The reciprocal space is divided into voxels $h_{v}, k_{v}, l_{v}$ of particular size $\delta h, \delta k, \delta l$. Intensities of pixels whose $h, k, l$ values are within $h_{v}+\delta h, k_{v}+\delta k, l_{v}+\delta l$ are averaged and the average value is assigned to the $h_{v}, k_{v}, l_{v}$ voxel. In other words, in this step we perform intensity averaging from all of the images to obtain the mean intensity of the $h_{v}+\delta h, k_{v}+\delta k, l_{v}+\delta l$ volume of reciprocal space. The maximum resolution of the reciprocal space map is achieved when the voxel size is close to the size of the pixel projected in reciprocal space. An example of a resulting reciprocal space map is shown in Fig. 11.

Ideally the choice of $\Delta l$ in equation (14) would be the voxel size $\delta l$ in the reciprocal space map. However, this is not always convenient because the reciprocal map might lack the in-plane resolution for proper integration. Therefore, in order to get inplane structure factors $F_{h k l}$, we may need to further average voxels in the range $\Delta l>\delta l$ for a given $h_{v}, k_{v}, l_{v}$, assuming a constant $F_{h k l}$ within $\Delta l . \Delta l$ is typically determined by the features in the diffraction rod. This step is in fact an in-plane projection and averaging of $F_{h k l}$ in the $\Delta l$ interval. Such projections for a crystal truncation rod (CTR) are shown in Fig. 12 for low and high $l$ values.

In most cases these projection images can now be integrated directly and, after applying the necessary corrections $\left(C_{\text {area }}\right.$, $\left.C_{\text {beam }}\right), F_{h k l}$ values can be calculated according to equation (14). The polarization corrections are applied to each pixel before voxelization.

Fig. 12(a) immediately shows one of the drawbacks for $F_{h k l}$ determination at low $l$ values. If the rocking scans are not performed with a small enough $\Delta \omega$ interval, some of the inplane voxels are not assigned any intensity value after the projection because the rod is almost tangent to the Ewald sphere (detector parallel to the rod). This can be overcome by interpolation or peak fitting as will be shown later. At higher $l$ values, the angle of the detector relative to the rod is higher, and therefore, for each image in the rocking scan, the Ewald sphere intersects the whole diameter of the rod and there are no unassigned voxels after the projection.

Background subtraction can be done in two ways: (i) on the projection images or (ii) from the side images of the rocking curve. In the first case the background is subtracted, as described in the previous section, by choosing the appropriate area on the projection image. In the second case we take the images from the rocking scan that do not intercept the rod (on

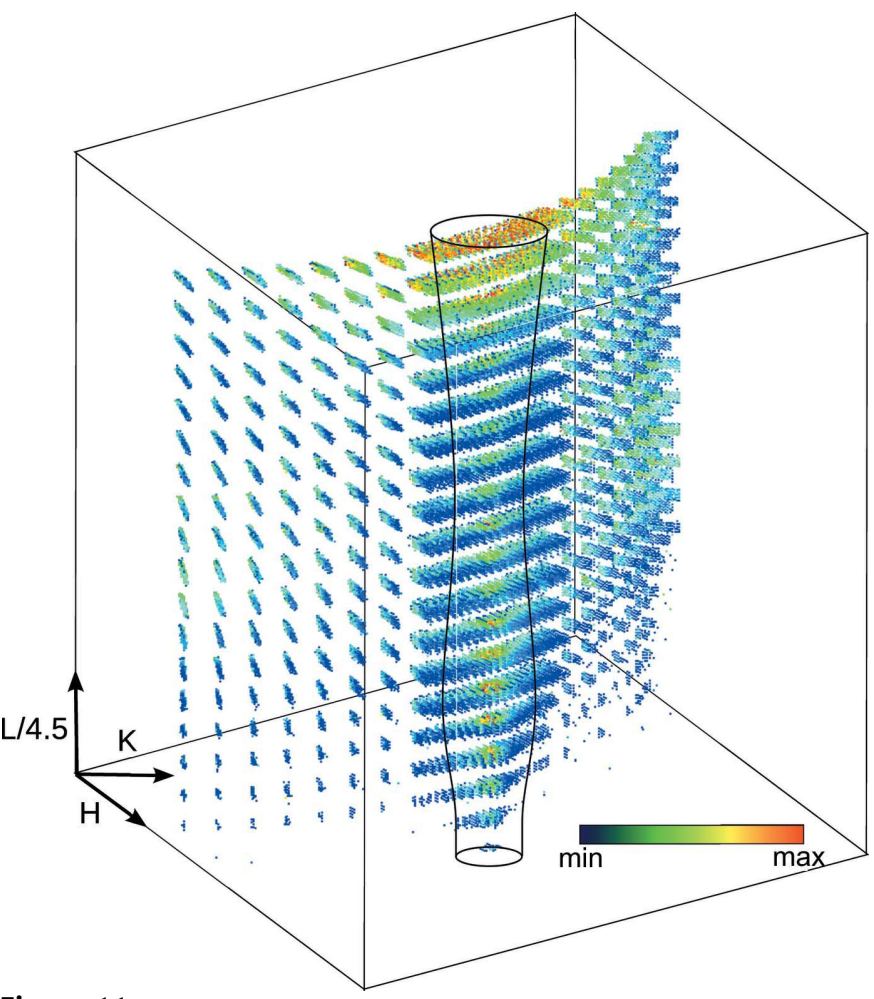

Figure 11

Reconstruction of a surface rod in reciprocal space $\left[\left(\frac{2}{3}, 0, l\right)\right.$ rod of a $\mathrm{CsO}$ layer adsorbed on $\operatorname{Pt}(111)]$. The reciprocal intensity map was reconstructed from rocking scans taken at different $l$. For this particular rod we used dense sampling in proximity to the rod (depicted as a deformed cylinder) and fewer images were taken in the background region. 

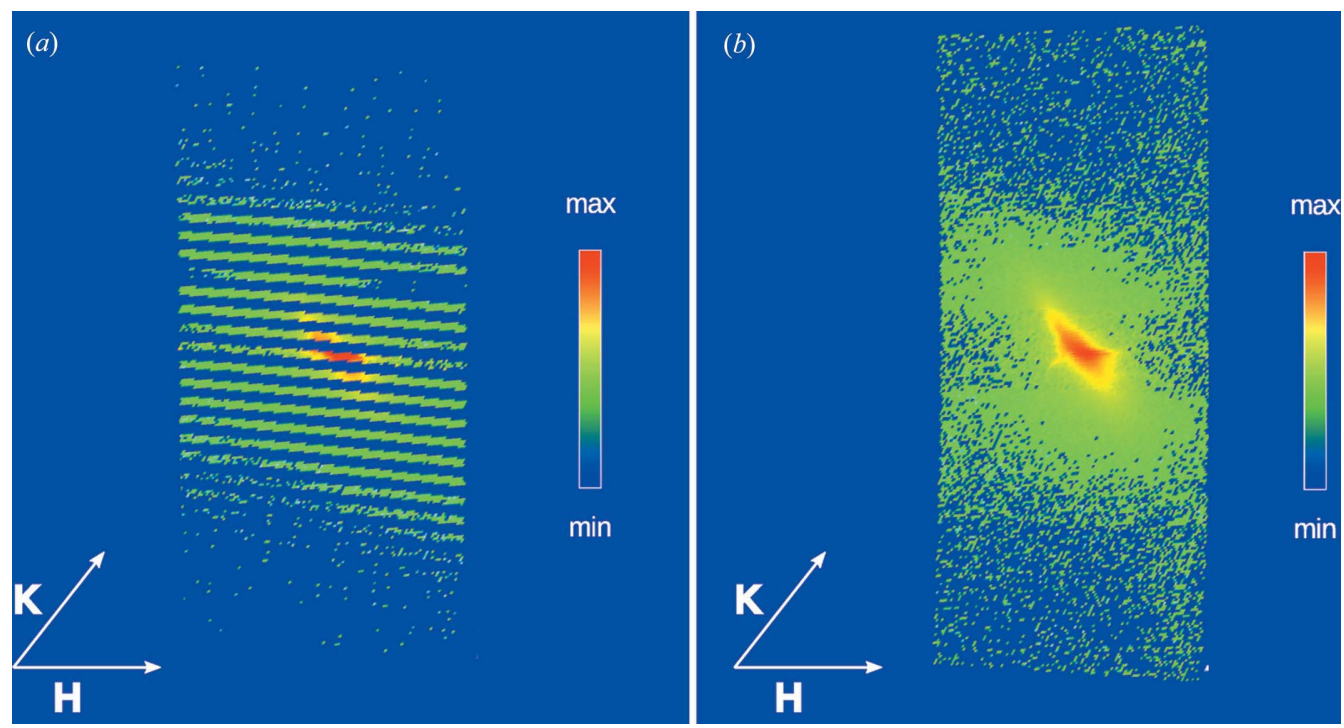

Figure 12

The projected intensity for a $(1,0, l)$ CTR with $l=0.45(a)$ and $l=2.65(b)$ [clean Pt(111), log scale].

Figure 13

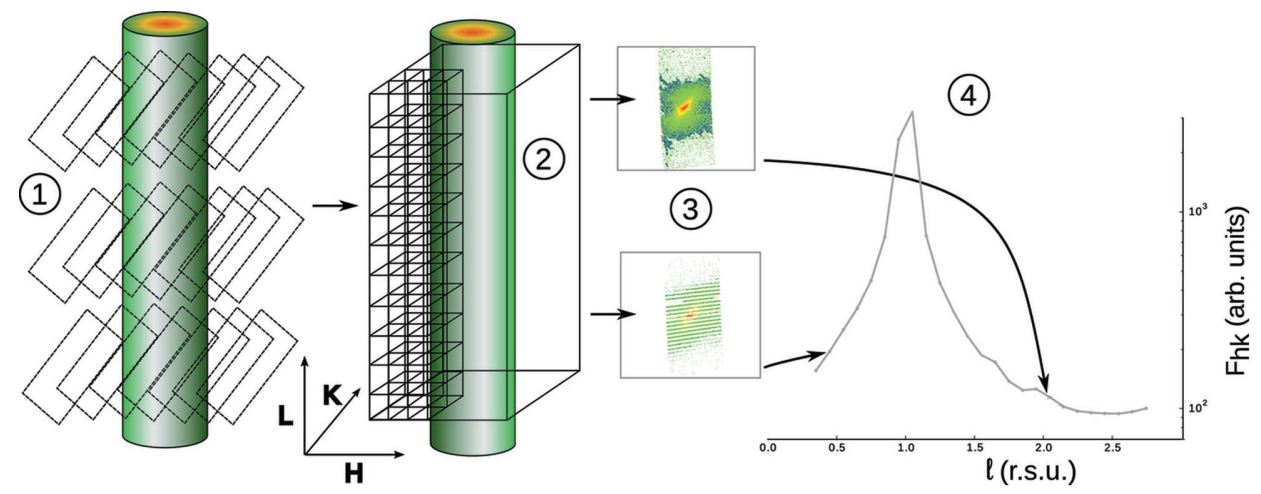

Summary of the integration in reciprocal space: rocking scans at various $l$ values are taken (1), a reciprocal space map is constructed (2), $l_{\text {const }}($ in-plane) projections are integrated or interpolated and then integrated (3), and $\left|F_{h k l}\right|$ is determined (4).

the side of the rod), average them and directly subtract them from all the images of the rocking scan. The projection is then made from background-subtracted images. This method has some inherent advantages. First, we have better background averaging as it is taken from multiple images. Second, the background originating from other parts of the experimental setup than the sample can also be easily subtracted. The main disadvantage is possible subtraction of other diffraction features (rings, Bragg peaks from the beryllium dome or misoriented crystallites) which interfere on the background images.

The summary of the analysis by integration in reciprocal space is depicted in Fig. 13:

(1) For a given $(h, k)$ rod, raw data are collected by performing rocking scans at different $l$ values.

(2) Each pixel on each image is assigned $h, k, l$ values of reciprocal space using the orientation matrix, and the polarization correction, $P$, is applied. Then the reciprocal space map is constructed.

(3) Voxels can be further averaged along the $l$ direction assuming $\partial\left|F_{h k l}\right| / \partial l \simeq 0$ and in-plane projection images are created.

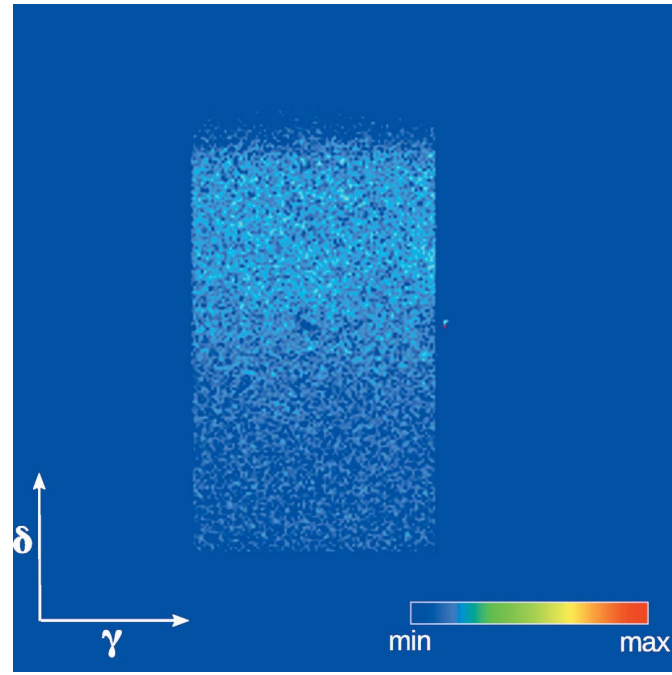

Figure 14

Image with the maximum intensity in the rocking curve $\left[\left(\frac{2}{3}, 0, l\right)\right.$ surface rod of a $\mathrm{CsO}$ layer on $\mathrm{Pt}(111)$ measured at $l=2.40]$. Intensity is in $\log$ scale. 
(4) $l_{\text {const }}$ (in-plane) projection images are either directly integrated or first interpolated and then integrated (see next section). The $\left|F_{h k l}\right|$ values are determined for a set of $l$ values.

\section{Detector acceptance problem}

As mentioned in the previous section, even in cases when we have incomplete information about the in-plane rod profile, it is possible to obtain correct $\left|F_{h k l}\right|$ values. Analytical methods of peak restoration for data acquired by a point detector have already been discussed at length (Robach et al., 2000) and further demonstrated on analysis of the $2 N$ reconstruction of the $\mathrm{Ge} / \mathrm{Si}(001)$ wetting layer (Zhou et al., 2011). For data acquired by two-dimensional detectors the reciprocal space integration approach is more appropriate, since we have a clearer view of the exact peak shape. It should be noted that the data restoration approaches are the last resort solution and proper data acquisition is strongly recommended.

Let us take as an example a surface reconstruction rod scattered from a CsO layer adsorbed on a $\mathrm{Pt}(111)$ surface. In the following we use a reciprocal lattice based on the surface $\mathrm{Pt}(111)$ unit cell. One image of the $\left(\frac{2}{3}, 0, l\right)$ rod interception at $l=2.40$ taken during the rocking scan is shown in Fig. 14 .

There are no particular features visible and the signal could be wrongly assigned as background. However, if we make a projection of the rocking scan, a wide rod is apparent (Fig. 15a). Even in this case, the active part of the detector does not completely intercept the whole diameter of the rod and some extrapolation method is needed to find the total intensity of the reflection.
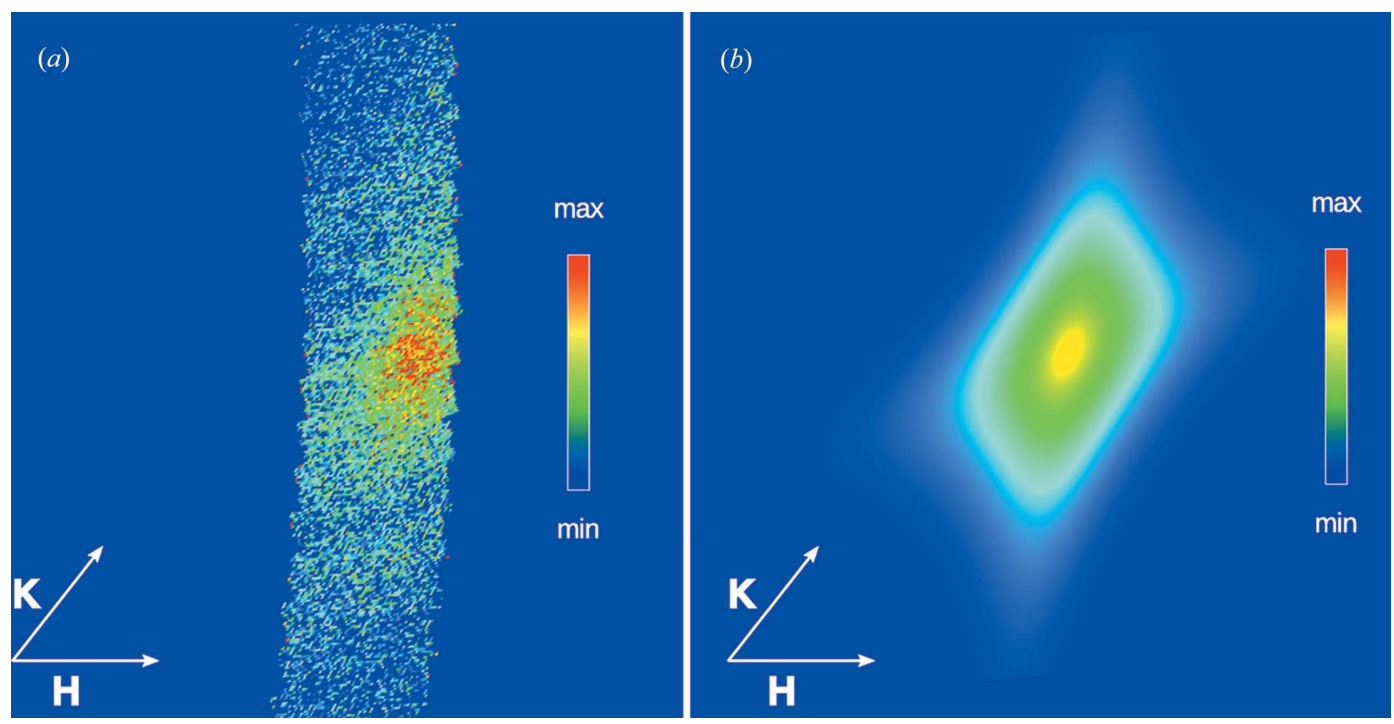

Figure 15

(a) The in-plane projection of the rocking curve $\left[\left(\frac{2}{3}, 0, l\right)\right.$ surface rod of a CsO layer on $\operatorname{Pt}(111)$ measured at $\left.l=2.40\right]$. (b) Lorentzian fit of the in-plane projection. Intensity is in log scale.
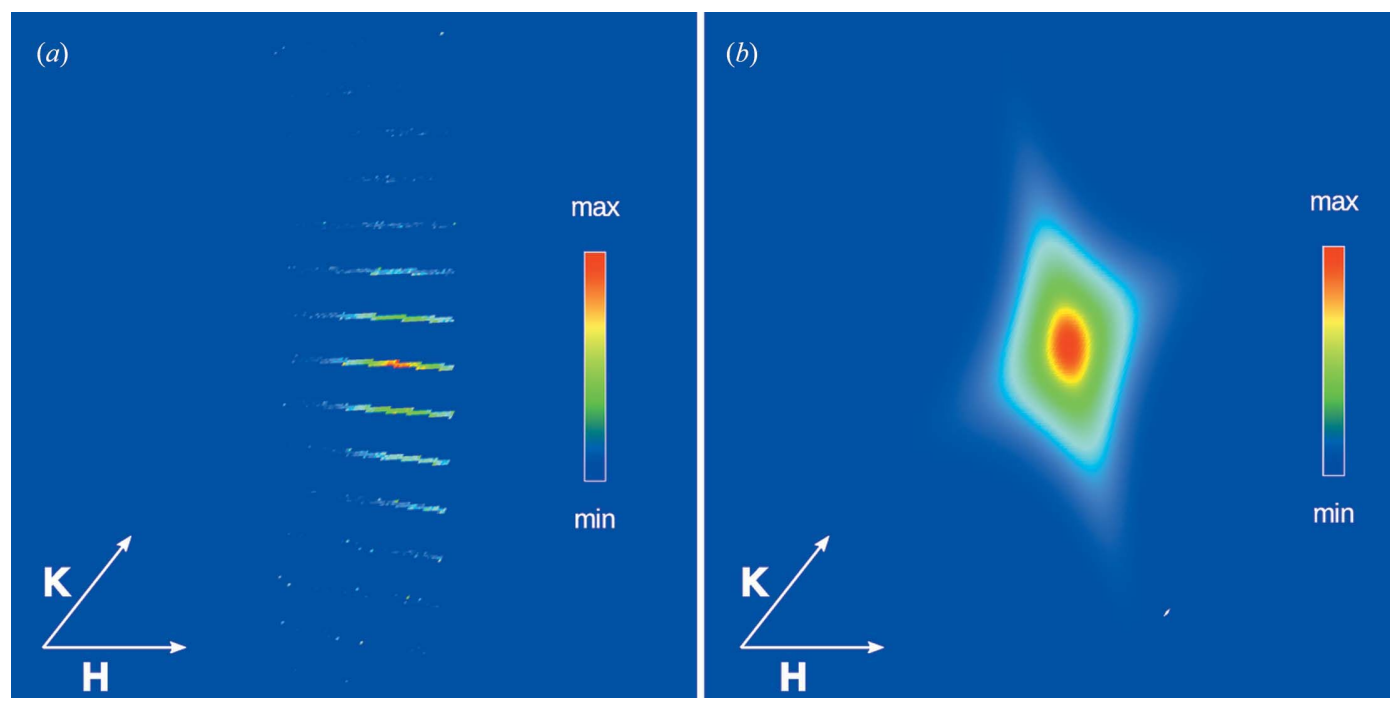

Figure 16

(a) The in-plane projection of the rocking curve $\left[\left(\frac{2}{3}, 0, l\right)\right.$ surface rod of a CsO layer on $\mathrm{Pt}(111)$ measured at $\left.l=0.45\right]$. (b) Lorentzian fit of the in-plane projection. Intensity is in log scale. 
In the example, the rod is fitted by a two-dimensional Lorentzian surface (Fig. 15b) and the intensity is calculated from the parameters of the fit. The same procedure can be applied for the projection of the same rod at $l=0.45$ (Fig. 16a), where the $\Delta \omega$ spacing between images taken during the rocking scan is insufficient to allow direct integration of the projection image.

Fig. 16(b) shows the Lorentzian two-dimensional fit that was used for intensity determination. It should be noted that fitting two-dimensional Lorentzian surfaces to incomplete projections is only one method and other interpolation/ extrapolation techniques can also be used.

Fig. 17 shows a comparison of different integration methods for a CTR. In this case the width of the rod intersection is smaller than the acceptance of the detector and the whole rod signal is contained in the detector for sufficiently high $l$ values.

The blue line in Fig. 17 shows the structure factors obtained by the direct integration of stationary scan images (\$3.1). The green curve is the reciprocal space integration of the rocking scan projection images (Fig. 12). The structure factors determined by fitting Lorentzian two-dimensional peaks on projected images and by simple interpolation of projected images are shown as red and violet curves, respectively. From the comparison we can directly conceive that the difference between curves is minimal for most of the $l$ values, as expected after proper treatment of the data. The main difference is at low $l$ values where the structure factor values are expected to be underestimated for non-extrapolated images and for direct integration. Interestingly, the difference at low $l$ values between fitted and extrapolated $\left|F_{h k l}\right|$ values is also quite large and in general depends on the quality of the interpolation algorithm. Small differences are also seen in the red curve. It is slightly shifted upwards as the CTR is not perfectly Lorentzian, and by fitting the Lorentzian shape the intensity is systematically overestimated. Also the bottom of the rod is flatter, but we did not observe the same rod flattening for

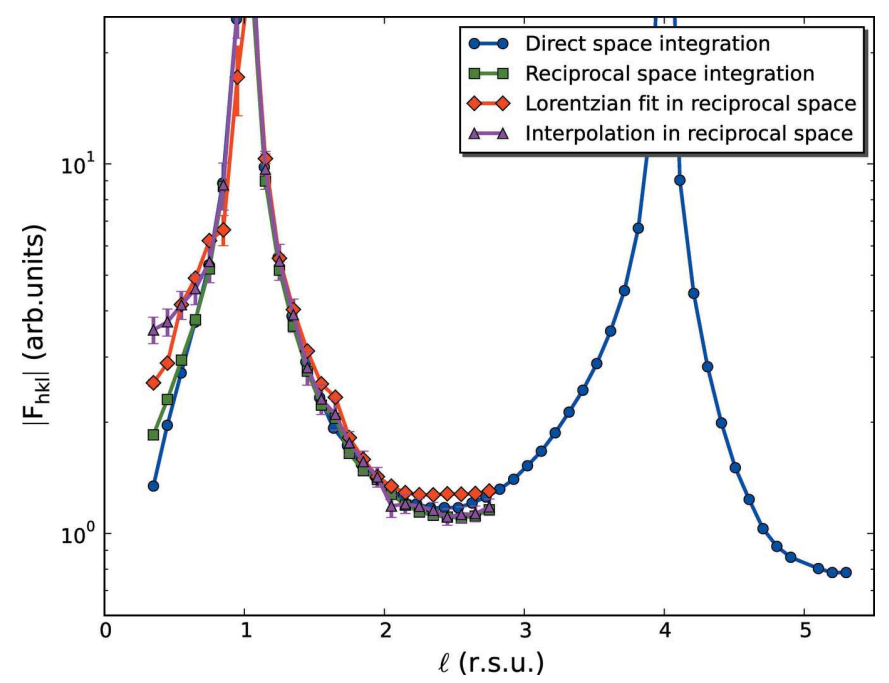

Figure 17

Comparison between different analysis methods: direct space integration, reciprocal space integration, Lorentzian fit in reciprocal space and interpolation in reciprocal space. $[(1,0, l)$ rod of clean $\mathrm{Pt}(111)$.] other rods measured on the same crystal. In conclusion, the Lorentzian fitting needs to be used with caution and perhaps a different mathematical description of the peak shape or interpolation can give better results.

The same comparison of different integration techniques is also shown for a surface rod with a large angular width (Fig. 18).

In this case the advantage of Lorentzian fitting is more obvious. Fig. 18 shows a comparison between the direct space integration of stationary scan images (blue curve), the reciprocal space integration of the rocking scan projection images (green curve) and two different interpolation methods in reciprocal space (red and violet). Because insufficient information has been obtained on the images during the stationary

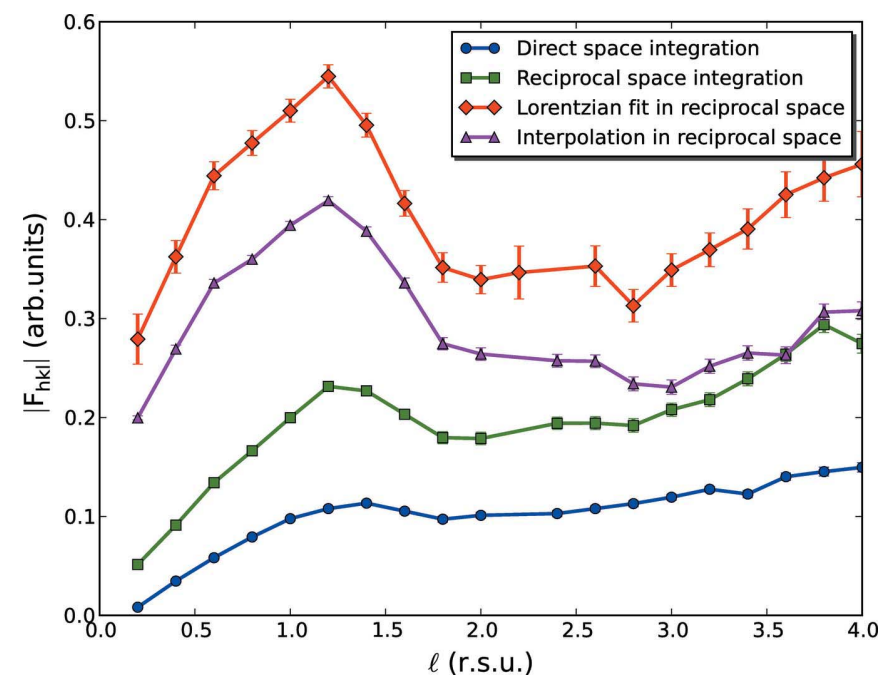

Figure 18

Comparison between different analysis methods when applied to a surface rod: direct space integration, reciprocal space integration, Lorentzian fit in reciprocal space and interpolation in reciprocal space. $\left[\left(\frac{2}{3}, 0, l\right)\right.$ rod of a CsO layer on $\mathrm{Pt}(111)$.]

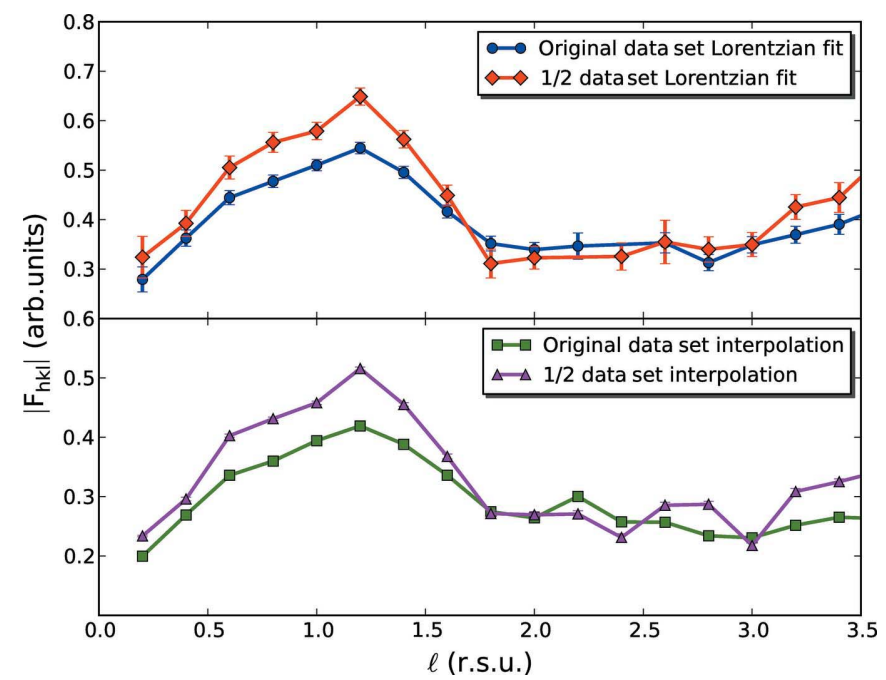

Figure 19

Comparison of Lorentzian fits (top) and interpolations (bottom) in reciprocal space when half of the total number of images in the rocking scan have been removed. $\left[\left(\frac{2}{3}, 0, l\right)\right.$ rod of a CsO layer on $\mathrm{Pt}(111)$.] 
Table 1

Comparison between different integration methods and data acquisition techniques.

\begin{tabular}{|c|c|c|c|c|}
\hline & Angular space integration & Reciprocal space integration & Stationary scan & Rocking scan \\
\hline Pros & Traditional approach, rapid implementation & Universal technique, Lorentzian corrections not needed & Fast acquisition & Low $l$ values \\
\hline Cons & $\begin{array}{l}\text { Lorentzian corrections need to be determined for each } \\
\text { diffractometer geometry and each type of scan }\end{array}$ & Computationally intensive & High $l$ values only & Slow acquisition \\
\hline
\end{tabular}

scan (Fig. 14), the direct space integration curve shows no particular modulation, and $F_{h k l}$ values obtained in this way can lead to a wrongly determined structure and should not be used. When we integrate projected intensities in reciprocal space (example in Fig. 15a), the curve has already a more reasonable profile (green curve). However, when we fit a twodimensional Lorentzian on projections or interpolate the images, the $F_{h k l}$ profile clearly shows modulation characteristic for a rod of a very thin layer (red and violet). The reason for the lack of modulation of the green curve is that the in-plane reciprocal mapping is incomplete, particularly at low $l$ values (Fig. 16), and interpolation is needed for proper integration.

The regularity of the Lorentzian fitting (top plot) and interpolation (bottom plot) is assessed in Fig. 19. First, all of the frames in the rocking scans were used to make the $h, k$ projections for fitting and interpolation (blue and green). Then, half of the images were removed from the data set (red and violet). As the number of the frames used in making the projections decreases, the fitting error increases because fewer voxels in reciprocal space are assigned an intensity. Nevertheless, the overall shape of the rod is still similar without any major differences. This figure points to the good reliability and robustness of the interpolation routines. We mainly use Lorentzian fitting or interpolation for rods that have large angular width or for measurements at low $l$ values where the in-plane reciprocal space mapping is not sufficient.

\section{Conclusions}

This article provides a framework to analyze surface X-ray diffraction data obtained with a two-dimensional detector. We show that the use of such a detector, together with a proper analysis method, can speed up the data acquisition significantly and provides accurate structure factors even at low $l$ values.

Two main approaches were proposed for the analysis. The first approach relies on angular space integration of twodimensional images, while the second is based on integration in reciprocal space. In most cases, stationary scans are sufficient to properly determine $\left|F_{h k l}\right|$. However, at low $l$ values, rocking scans are necessary for correct integration. On the basis of the above, the best approach is to take rocking scans for low $l$ values and a stationary scan for higher $l$ values for each diffraction rod.
Analysis methods of such scans were proposed in $\$ \S 3.2$ and 4. If the detector acceptance is smaller than the width of a reflection, extrapolation of reciprocal space map projections gives accurate $\left|F_{h k l}\right|$ values (\$5). Although integration in reciprocal space is a more universal approach, the integration in direct space is sufficient in many cases and does not require extensive programming and computational power. A summary is given in Table 1.

JD would like to thank Vincent Armando Sole for providing reciprocal mapping Python code. TZ would like to thank Vincent Favre Nicolin and Odile Robach for useful discussions, and Nils Blanc and Fabien Jean for providing some of the experimental data.

\section{References}

Cowley, J. M. (1975). Diffraction Physics. Amsterdam: North Holland.

Feidenhans'l, R. (1989). Surf. Sci. Rep. 10, 105-188.

Kriegner, D., Wintersberger, E. \& Stangl, J. (2013). J. Appl. Cryst. 46, 1162-1170.

Mariager, S. O., Lauridsen, S. L., Dohn, A., Bovet, N., Sørensen, C. B., Schlepütz, C. M., Willmott, P. R. \& Feidenhans'l, R. (2009). J. Appl. Cryst. 42, 369-375.

Robach, O., Garreau, Y., Aïd, K. \& Véron-Jolliot, M. (2000). J. Appl. Cryst. 33, 1006-1018.

Robinson, I. (1990). Handbook on Synchrotron Radiation, Vol III. Amsterdam: Elsevier.

Robinson, I. \& Tweet, D. (1992). Rep. Prog. Phys. 55, 599-651.

Schlepütz, C. M., Herger, R., Willmott, P. R., Patterson, B. D., Bunk, O., Brönnimann, Ch., Henrich, B., Hülsen, G. \& Eikenberry, E. F. (2005). Acta Cryst. A61, 418-425.

Schlepütz, C. M., Mariager, S. O., Pauli, S. A., Feidenhans'l, R. \& Willmott, P. R. (2011). J. Appl. Cryst. 44, 73-83.

Serra, J. (1982). Image Analysis and Mathematical Morphology, Vol. 1. London: Academic Press.

Sjölin, 1. \& Wlodawer, A. (1981). Acta Cryst. A37, 594-604.

Solé, V. A., Papillon, E., Cotte, M., Walter, Ph. \& Susini, J. A (2007). Spectrochim. Acta Part B, 62, 63-68.

Specht, E. \& Walker, F. (1993). Phys. Rev. B, 47, 13743-13751.

Vlieg, E. (1997). J. Appl. Cryst. 30, 532-543.

Vlieg, E. (2012). Surface and Interface Science, Vol. 1, Concepts and Methods. Weinheim: Wiley.

Vlieg, E. \& Robinson, I. (1992). Synchrotron Radiation Crystallography. London: Academic Press.

Zhou, T., Renaud, G., Revenant, C., Issartel, J., Schuelli, T. U., Felici, R. \& Malachias, A. (2011). Phys. Rev. B, 83, 195426. 